\title{
SEMI-ANALYTICAL SOLUTION OF OPTIMIZATION ON MOON-POOL SHAPED WEC
}

\author{
W.C. ZHANG, \\ H.X. Liu, \\ X.W.ZHANG, \\ L. ZHANG \\ College of Shipbuilding Engineering, Harbin Engineering University, Harbin,China
}

\begin{abstract}
In order to effectively extract and maximize the energy from ocean waves, a new kind of oscillating-body WEC (wave energy converter) with moon pool has been put forward. The main emphasis in this paper is placed on inserting the damping into the equation of heaving motion applied for a complex wave energy converter and expressions for velocity potential added mass, damping coefficients associated with exciting forces were derived by using eigenfunction expansion matching method. By using surface-wave hydrodynamics, the exact theoretical conditions were solved to allow the maximum energy to be absorbed from regular waves. To optimize the ability of the wave energy conversion, oscillating system models under different radius-ratios are calculated and comparatively analyzed. Numerical calculations indicated that the capture width reaches the maximum in the vicinity of the natural frequency and the new kind of oscillating-body WEC has a positive ability of wave energy conversion.
\end{abstract}

Keywords: wave energy; eigenfunction expansion; moon pool; conversion ability; optimization

\section{INTRODUCTION}

With worldwide demand for energy and electricity unabated, over the past decades, so much considerable attention and effort have been devoted to the problem of extracting energy from the ocean waves and to investigating theoretically and experimentally several types of devices in order to maximize their efficiency. The major concern in extensively today is to continue to improve the oscillating bodies and the oscillating water columns which are the two main types of devices containing the oscillating system and PTO (power take-off) mechanism.

Oscillating-body WEC reflects a simple but robust technology, especially for the point absorbing WEC, which consists of buoys or floating bodies to capture the waves heaving motion. Oscillating-body WEC can take advantage of the body with resonant and periodic motion caused by the ocean waves with large forces at slow speeds to capture energy. Comparing to other types of WECs, the oscillating body is usually small in size and often used in arrays. Due to the immense potential and prospect of wave energy technology, a number of wave conversion devices have been designed and analyzed by researchers. Budal K et al [1] developed a kind of buoy, consisting of a spherical floater which could perform heaving oscillations relative to a strut connected to an anchor on the sea bed through a universal joint and the buoy could be phase-controlled by latching and was equipped with an air turbine. Weinstein et al. [13] outlined a project of developing the Makah Bay pilot offshore power plant, which used Aqua Energy's point absorber wave energy conversion device Aqua 
BUOY. The device represented the next generation of the technology that combines the Swedish Hose-Pump and the IPS Buoy technologies to generate clean energy from ocean waves. Margheritini et al. [9] described concept of the sea slot-cone generator (SSG) WEC and the studies behind the process that leaded to the construction of such wave energy conversion system. There has been a turning point in structure with moon-pool in accordance with the advent of extracting energy from the ocean waves. In the present work, we focus on need for a new kind of oscillating-body WEC which is cylindrical structures with a moon pool with a restricted entrance associated with a smaller disk inner.

To being with we will provide a brief background on the study of waves around cylindrical cylinders. The pioneer work on the semi-analytical solution of the oscillating system can be traced to MacCamy and Fuchs [8] on the simplest case of a vertical cylinder standing on the seabed. Since Miles and Gilbert [11] formulated the problem of the scattering of the surface waves by a circular dock and obtained a variational approximation to the far filed, a number of works have been done for different types of vertical cylinders (truncated and composed of different diameters) by using so called eigenfunction matching method which consists of dividing the fluid domain into several cylindrical subdomains and matching the eigenfunction expansions in different subdomain. Many research studies have been carried out on this topic such as Liu et al. [7] for a series of different configurations of a single cylinder. The central issue in all above studies is the analytical method based on the series expansion of eigenfunctions which satisfy Laplace equation and are used to evaluate the velocity potentials around cylindrical structures and to compute the wave loads. The fluid domain around the complex structure hull is divided into an army of cylindrical subdomains. In each domain, the different expansions of eigenfunctions are developed and the unknown coefficients of expansion series are determined by the boundary condition on cylinder hull and the continuous conditions through the control surfaces which separate the domains.

The cylindrical structure with moon pool which contains resonance frequency is developed by Garrett [5], a bottomless cylinder which is equivalent to a cylinder with fully opened moon pool. Mavrakos and Chatjigeorgiou [10] have solved wave loads on a stationary floating bottomless cylinder with a finite wall thickness. Very recently, Chen et al. [2] introduced the free surface dissipation for wave diffraction of cylindrical structures with moon pool of restricted entrance using the semi-analytical solution and the positive agreement of the free-surface elevation on the center of moon pool with measurements in model test is indicative of the instructional significance to engineering design.

An additional difficulty is related to the conception of the PTO which should allow the production of usable energy. Considerable efforts have been made by several authors to investigate the performance of the point absorber WEC with a linear damping. Eriksson et al. [3] investigated the coupling behavior of the linear generator and the floating buoy in the frequency domain. Price et al. [12] studied the capture width of WECs in detail and discusses the effects of the PTO control on the capture width. Yeung et al. [16] utilizes both the theoretical and mathematical models to obtain the hydrodynamics properties of the floating buoy and studies the performance of the UC-Berkeley WEC via experimental approach. Special attention was paid on power take-off performance and design, and on the control of the system. Henderson [6] described the hydraulic power takeoff system employed in the Pelamis WEC and the process of the systems development was also presented, including simulation and laboratory tests at $1 / 7$ th and full scale. Falcao [4] presented a general method of modeling oscillating-body WECs with hydraulic power take-off and gas accumulator. With the assumption of linear PTO and linear surfacewave hydrodynamics, Wu et al. [14-15] derived a reciprocal relationship between the spring stiffness, damping coefficient and diffraction properties, and a general expression for timeaveraged power output was also presented.
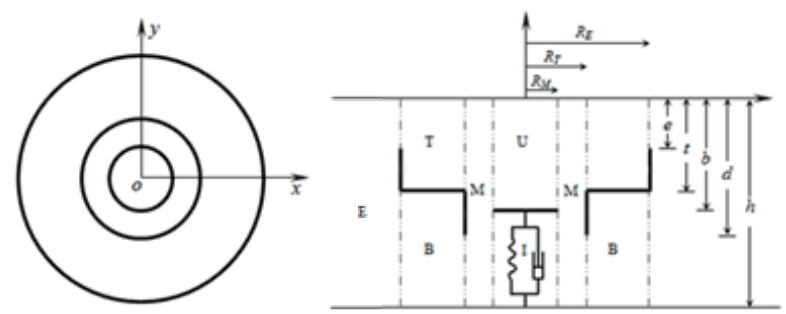

Fig. 1 Cylindrical structures with a moon-pool with a restricted entrance associated with smaller disk inner

This paper focuses on the performance analysis and geometrical optimizations of a newly proposed two-body oscillating wave energy converter which contains a submerged moon-pool shaped paddock and a coaxial disk. The damper installed on the paddock and activated by the bodies' oscillations contains a linear spring and controllable velocity dependent impedance. The plane wave theory based upon the eigenfunction matching method considering the complex boundary conditions is used for the hydrodynamic analysis of the structures in frequency domain. The expressions of the diffracted and radiated velocity potentials in each subdomain are analytically established. The linear wave excitation forces and hydrodynamic coefficients are further obtained. Finally, the numerical results of the wave forces, hydrodynamic coefficients and captured width radios are illustrated considering different geometries of the paddocks.

\section{METHODS}

The oscillating-body WEC considered in this work is shown in Fig.1. At the water depth of e, a barrel shaped structure of radius $R_{E}$ with a moon pool of radius $R_{T}$ at the bottom center is submerged vertically in the fluid of constant depth $\mathrm{h}$. The heights of the barrel and moon pool are denoted by $(\mathrm{t}-\mathrm{e})$ and (b-e) respectively. A circular quality disk (the oscillating body) of smaller radius $R_{M}$ is assumed to be installed vertically and coaxially with the moon pool under the undisturbed free surface of $\mathrm{d}$. It is further assumed that a bottom-mounted 
nonlinear PTO device is installed at the bottom of the circular disk for energy extraction, and the thicknesses of the barrel, the moon pool wall and the circular plate are all thin and are set to zero in developing the theory.

We defined the cylindrical coordinated system $(\mathrm{r}, \theta, \mathrm{z})$ by its origin located at the center of the cylinder and on the mean plane of the free surface. The axis oz is vertically upward. By assuming that the fluid is perfect and flow irrotational, fluid velocity $\mathrm{V}(\mathrm{r}, \theta, \mathrm{z}, \mathrm{t})$ can be represented by the gradient of a potential function $\Psi(\mathrm{r}, \theta, \mathrm{z}, \mathrm{t})$ which satisfies the Laplace's equation:

$$
\mathbf{V}(r, \theta, z, t)=\nabla \Psi(r, \theta, z, t) \text { with } \nabla^{2} \Psi(r, \theta, z, t)=0
$$

in the fluid domain. Considering a linear surface wave of small amplitude A propagates along the positive $\mathrm{x}$-axis direction, given the above assumptions, the flow field can be described by a velocity potential:

$$
\Psi(r, \theta, z, t)=\mathfrak{R}\left\{\Phi(r, \theta, z) e^{-i \omega t}\right\}
$$

On the mean free surface $\mathrm{z}=0$, the combined kinematic and dynamic boundary condition is given by:

$$
-f^{2} \Phi+\partial_{z} \Phi=0
$$

in which $f^{2}=\omega^{2} / g$ with $\omega$ as wave frequency and $g$ the acceleration due to gravity.

On the hull is expressed by:

$$
\partial_{n} \Phi=V_{n}
$$

where $\mathrm{V}_{\mathrm{n}}$ is the cylinder's velocity in the direction normal to the hull. In the same way, the boundary condition on the sea bed is $\mathrm{z}=-\mathrm{h}$ is $\partial_{2} \Phi=0$. In addition, we have to a radiation condition requiring that all perturbation due to cylinders disappears at infinity, i.e.

$$
\Phi \rightarrow \Phi_{0} \text { at } r \rightarrow \infty
$$

to ensure the uniqueness of the solution, where $\Phi_{0}$ representing the velocity of incoming waves is given as:

$$
\Phi_{0}=-A g / \omega \cdot \cosh k_{0}(z+h) / \cosh k_{0} h \cdot \exp \left(i k_{0} r \cos \theta\right)
$$

in which $\left(\mathrm{A}, \omega, \mathrm{k}_{0}\right)$ stand for the amplitude, frequency and wavenumber, respectively, the wave number $\mathrm{k}_{0}$ defined by $\mathrm{k}_{0}$ and $\mathrm{k}_{0} \mathrm{~h}=f^{2}$ incoming waves propagating along the positive $\mathrm{x}$-axis.

\section{POWER TAKE-OFF MECHANISM}

In order to calculate the optimal extraction efficiency of the WEC, we consider the simple case of the body with a singledegree of freedom, oscillating in heave. To know the optimal relationship between the quadratic PTO device and key hydrodynamic parameters, a linear PTO and iterative method will be introduced. Given the flow field environment of the oscillating body, the governing equation can be condensed into the following expression:

$$
m \ddot{Z}=F_{d}+F_{r}+F_{m}
$$

where $\mathrm{m}$ is the proper mass of the oscillating body, $\mathrm{Z}$ is the displacement and the dot denotes the differential of time t. $F_{d}$ is the vertical force produced by the incident waves on the assumedly fixed body (excitation or diffraction force), $\mathrm{F}_{\mathrm{r}}$ is the hydrodynamic force due to the body oscillation in otherwise calm water (radiation force), $\mathrm{F}_{\mathrm{m}}$ is the vertical component of the force applied on the oscillating body by the PTO mechanism.

In the case of regular waves of frequency $\omega$, the excitation force $\mathrm{F}_{\mathrm{D}}$ and displacement $\mathrm{Z}$ are both simple harmonic functions of time $t$ and for convenience, we can write $\mathrm{Z}(\mathrm{t})=\mathrm{ze}^{-\mathrm{i} \omega \mathrm{t}}$ and $\mathrm{F}_{\mathrm{d}}(\mathrm{t})=f_{\mathrm{d}} \mathrm{e}^{-\mathrm{i} \omega \mathrm{t}}$. Here, $\mathrm{z}$ and $\mathrm{f}_{\mathrm{d}}$ are the complex amplitudes respectively. It is convenient to decompose the radiation force as inertial force and wave making damping, which can be expressed as $F_{r}=\mu \ddot{Z}-\lambda \dot{Z}$. Here $\mu$ is the added mass and $\lambda$ is the radiation damping coefficient ( $\lambda$ cannot be negative according to physical reasons). The PTO mechanism force $\mathrm{F}_{\mathrm{m}}$ contains spring restoring force and damping which is associated with the energy extraction and for linear damping can be expressed as $\mathrm{F}_{\mathrm{m}}=-\mathrm{kZ}-\mathrm{cZ}$. Here $\mathrm{k}$ is the spring rigidity coefficient and $\mathrm{c}$ is the damping coefficient.

For bodies with a vertical axis of symmetry oscillating in heave, by combining the expressions of the above forces, we find that $\mathrm{z}=f_{\mathrm{d}} /\left[-\omega^{2}(\mathrm{~m}+\mu)-\mathrm{i} \omega(\lambda+\mathrm{c})+\mathrm{k}\right]$. Based upon the governing equation of the oscillating body in frequency domain, the time-averaged absorbed power can be calculated and given as:

$$
\bar{P}=c / 2 \cdot f_{d} f_{d}^{*} /\left\{[k / \omega-\omega(m+\mu)]^{2}+(\lambda+c)^{2}\right\}
$$

where the asterisk denotes the conjugate. This show that, for a given body and given incident wave (i.e., fixed $\omega, \lambda$ and $f_{d}$ ), the time-averaged absorbed power will reach the peak when the values of $\mathrm{k}$ and $\mathrm{c}$ satisfy $\overline{\partial_{\mathrm{k}} \mathrm{P}}=\partial_{c} \mathrm{P}=0$. Combining the Eq. (12), we have $\mathrm{k}_{\text {opt }}=\omega^{2}(\mathrm{~m}+\lambda), \mathrm{c}_{\mathrm{opt}}=|\lambda|$. Here $\mathrm{k}_{\mathrm{opt}}$ and $\mathrm{c}_{\text {opt }}$ are the optimum PTO coefficients. Furthermore, we will get the maximum time averaged absorbed power $\mathrm{P}_{\max }=f_{\mathrm{d}} f_{\mathrm{d}}^{\star}$ / $8|\lambda|$. In order to calculate the power take-off efficiency $\eta$, we need to know the time-power in unite wavelength and in the width of the oscillating body, which can be expressed as:

$$
\bar{P}_{0}=\rho g^{2} A^{2} R_{m} / 2 \omega \cdot\left(2 k_{0} h+\sinh 2 k_{0} h\right) /\left(1+\cosh 2 k_{0} h\right)
$$

Then the maximum power take-off efficiency $\eta$ of the WEC can be obtained:

$\eta=\bar{P}_{\max } / \bar{P}_{0}=\omega f_{d} f_{d}^{*} /\left(4|\lambda| \rho g^{2} A^{2} R_{m}\right) \cdot\left(1+\cosh 2 k_{0} h\right) /\left(2 k_{0} h+\sinh 2 k_{0} h\right)$

\section{SEMI-ANALYTICAL SOLUTION OF HYDRODYNAMIC} COEFFICIENTS

In order to obtain semi-analytical solution of hydrodynamic coefficients $\mu, \lambda$ and $f_{d}$, the diffraction velocity potential $\Phi_{7}$ 
and radiation velocity potential for heave mode $\Phi_{3}$ around a new kind of oscillating-body WEC can be expressed as:

$$
\begin{gathered}
\Phi_{7}(r, \theta, z)=-\frac{A g}{\omega} \sum_{l=0}^{\infty} \phi_{7}(r, z) \cos l \theta \\
\Phi_{3}(r, \theta, z)=\phi_{3}(r, z)
\end{gathered}
$$

The appropriate expression of $\phi(\mathrm{r}, \mathrm{z})$ composed of eigenfunctions should be obtained by considering the boundary conditions in a cylindrical domain of fluid. The fluid domain surrounding structures is divided into six cylindrical subdomains as shown in Fig.1. In such case, the diffraction velocity potential $\Phi_{7}$ and radiation velocity potential for heave mode $\Phi_{3}$ around the structure in each of the aforementioned subdomains, namely, $\Phi_{7,3 \mathrm{E}}$ in the exterior subdomain $\mathrm{E}, \Phi_{7,3 \mathrm{~T}}$ in the above subdomain T, $\Phi_{7,3 \mathrm{~B}}$ in the underneath subdomain $\mathrm{B}, \Phi_{7,3 \mathrm{M}}$ in the middle subdomain $\mathrm{M}, \Phi_{7,3 \mathrm{U}}$ in the inner above subdomain $\mathrm{U}, \Phi_{7,3 \mathrm{~L}}$ in the inner underneath subdomain L. Following Garrett (1970), the velocity potential in the exterior domain E is written as:

$$
\begin{gathered}
\phi_{7 E}^{l}(r, z)=a_{0}^{l} Z_{0}\left(k_{0}, z, h\right) \mathbf{H}_{l}\left(k_{0} r\right)+\sum_{n=1}^{\infty} a_{n}^{l} Z_{n}\left(k_{0}, z, h\right) \mathbf{H}_{l}\left(k_{n} r\right)+\phi_{0}^{l}(r, z) \\
\phi_{3 E}(r, z)=\phi_{7 E}^{0}(r, z)
\end{gathered}
$$

with $\mathrm{Z}_{\mathrm{n}}\left(\mathrm{k}_{\mathrm{n}}, \mathrm{z}, \mathrm{h}\right)=\cos \mathrm{k}_{\mathrm{n}}(\mathrm{z}+\mathrm{h}) /\left(2 \mathrm{k}_{\mathrm{n}} \mathrm{h}+\sin 2 \mathrm{k}_{\mathrm{n}} \mathrm{h}\right)$ and $\mathrm{k}_{\mathrm{n}}$ defined by $\mathrm{k}_{\mathrm{n}} \tan \mathrm{k}_{\mathrm{n}} \mathrm{h}=-\mathrm{f}^{2}$ for $\mathrm{n} \geq 1$. In the above subdomain $\mathrm{T}$, the velocity potential $\phi_{\mathrm{T}}^{1}$ is written as:

$$
\begin{gathered}
\phi_{7 T}^{l}(r, z)=b_{0}^{l} Z_{0}\left(\gamma_{0}, z, d\right) \mathbf{J}_{l}\left(\gamma_{0} r\right)+\sum_{n=1}^{\infty} b_{n}^{l} Z_{n}\left(\gamma_{n}, z, d\right) \mathbf{I}_{l}\left(\gamma_{n} r\right)+\tilde{b}_{0}^{l} Z_{0}\left(\gamma_{0}, z, d\right) \mathbf{H}_{l}\left(\gamma_{0} r\right)+\sum_{n=1}^{\infty} \tilde{b}_{n}^{l} Z_{n}\left(\gamma_{n}, z, d\right) \mathbf{K}_{l}\left(\gamma_{n} r\right) \\
\phi_{3 T}(r, z)=\phi_{7 T}^{0}(r, z)+\left(z \omega^{2}+g\right) / \omega^{2}
\end{gathered}
$$

with the wave number defined by $\gamma_{0} \tanh \gamma_{0} \mathrm{t}=\mathrm{f}^{2}$ and $\gamma_{\mathrm{n}} \tan \gamma_{\mathrm{n}} \mathrm{t}=-\mathrm{f}^{2}$ for $\mathrm{n} \geq 1$. In the subdomain $\mathrm{B}$, the velocity potential $\phi_{\mathrm{B}}^{1}$ can be expressed by:

$$
\begin{gathered}
\phi_{7 B}^{l}(r, z)=c_{0}^{l} P_{0}^{l}(r)+\sum_{n=1}^{\infty} c_{n}^{l} \cos \lambda_{n}(z+h)\left[\mathbf{K}_{I}^{n l} \cdot \mathbf{I}_{l}\left(\lambda_{n} r\right)-\mathbf{I}_{I}^{n l} \cdot \mathbf{K}_{l}\left(\lambda_{n} r\right)\right]+\tilde{c}_{0}^{l} \tilde{P}_{0}^{l}(r)+\sum_{n=1}^{\infty} \tilde{c}_{n}^{l} \cos \lambda_{n}(z+h)\left[\mathbf{I}_{E}^{n l} \cdot \mathbf{K}_{l}\left(\lambda_{n} r\right)-\mathbf{K}_{E}^{n l} \cdot \mathbf{I}_{l}\left(\lambda_{n} r\right)\right] \\
\phi_{3 B}(r, z)=\phi_{7 B}^{0}(r, z)+\left[2(z+h)^{2}-r^{2}\right] /[4(h-t)]
\end{gathered}
$$

in which the function $\mathrm{P}_{0}^{\mathrm{l}}$ and $\overline{\mathrm{P}}_{0}^{\mathrm{l}}$ are defined by

$$
\begin{gathered}
P_{0}^{0}(r)=\ln \left(r / R_{I}\right) / \ln \left(R_{E} / R_{I}\right), \tilde{P}_{0}^{0}(r)=\ln \left(R_{E} / r\right) / \ln \left(R_{E} / R_{I}\right) \text { with } l=0 \\
P_{0}^{l}(r)=\left[\left(r / R_{I}\right)^{l}-\left(R_{I} / r\right)^{l}\right] /\left[\left(R_{E} / R_{I}\right)^{l}-\left(R_{I} / R_{E}\right)^{l}\right], \tilde{P}_{0}^{l}(r)=\left[\left(R_{E} / r\right)^{l}-\left(r / R_{E}\right)^{l}\right] /\left[\left(R_{E} / R_{I}\right)^{l}-\left(R_{I} / R_{E}\right)^{l}\right] \text { with } l>0
\end{gathered}
$$

The constants $\left\{\mathbf{K}_{I}^{n l}, \mathbf{I}_{I}^{n l}, \mathbf{K}_{E}^{n l}, \mathbf{I}_{E}^{n l}\right\}$ are given by:

$$
\begin{aligned}
\left\{\mathbf{K}_{I}^{n l}, \mathbf{I}_{I}^{n l}, \mathbf{K}_{E}^{n l}, \mathbf{I}_{E}^{n l}\right\}=\left\{\mathbf{K}_{l}\left(\lambda_{n} R_{I}\right), \mathbf{I}_{l}\left(\lambda_{n} R_{I}\right), \mathbf{K}_{l}\left(\lambda_{n} R_{E}\right), \mathbf{I}_{l}\left(\lambda_{n} R_{E}\right)\right\} / C_{I E}^{n l} & \\
C_{I E}^{n l}=\mathbf{K}_{l}\left(\lambda_{n} R_{I}\right) \mathbf{I}_{l}\left(\lambda_{n} R_{E}\right)-\mathbf{K}_{l}\left(\lambda_{n} R_{E}\right) \mathbf{I}_{l}\left(\lambda_{n} R_{I}\right) & \text { with } \mathrm{n} \geq 1 \text { and } 1 \geq 0
\end{aligned}
$$

in which, the wave number $\lambda_{n}$ is defined by $\lambda_{n}=n \pi /(h-t)$ to satisfy the boundary condition $\partial_{z} \phi_{B}^{1}=0$ on both surfaces $\mathrm{z}=-\mathrm{h}$ and $\mathrm{z}=-\mathrm{t}$. In the middle subdomain $\mathrm{M}$, the velocity potential $\phi_{\mathrm{M}}^{1}$ can be expressed by:

$$
\begin{gathered}
\phi_{7 M}^{l}(r, z)=d_{0}^{l} Z_{0}\left(k_{0}, z, h\right) \mathbf{J}_{l}\left(k_{0} r\right)+\sum_{n=1}^{\infty} d_{n}^{l} Z_{n}\left(k_{n}, z, h\right) \mathbf{I}_{l}\left(k_{n} r\right)+\tilde{d}_{0}^{l} Z_{0}\left(k_{0}, z, h\right) \mathbf{H}_{l}\left(k_{0} r\right)+\sum_{n=1}^{\infty} \tilde{d}_{n}^{l} Z_{n}\left(k_{n}, z, h\right) \mathbf{K}_{l}\left(k_{n} r\right) n \geq 1 \\
\phi_{3 M}(r, z)=\phi_{7 M}^{0}(r, z)
\end{gathered}
$$

In the inner above $U$, the velocity potential $\phi_{U}^{1}$ can be expressed by:

$$
\begin{gathered}
\phi_{7 L}^{l}(r, z)=f_{0}^{l}(r / R)^{l}+\sum_{n=1}^{\infty} f_{n}^{l} \cos \lambda_{n}(z+h) \mathbf{I}_{l}\left(\lambda_{n} r\right) \\
\phi_{3 L}(r, z)=\phi_{7 L}^{0}(r, z)+\left[2(z+h)^{2}-r^{2}\right] /[4(h-d)]
\end{gathered}
$$

In the inner underneath $\mathrm{I}$, the velocity potential $\phi_{\mathrm{I}}^{1}$ can be expressed by:

$$
\begin{gathered}
\phi_{7 L}^{l}(r, z)=f_{0}^{l}(r / R)^{l}+\sum_{n=1}^{\infty} f_{n}^{l} \cos \lambda_{n}(z+h) \mathbf{I}_{l}\left(\lambda_{n} r\right) \\
\phi_{3 L}(r, z)=\phi_{7 L}^{0}(r, z)+\left[2(z+h)^{2}-r^{2}\right] /[4(h-d)]
\end{gathered}
$$

for $n \geq 1$. In the eigenfunction expansion for the velocity potentials, the unknown coefficients are to be determined by taking advantage of the orthogonality, in the so-called Garrett's method (Garrett,1971), according to matching the potential and its normal derivative on the juncture boundaries surfaces shared by subdomains. Once the velocity potential is known, the diffraction wave force and hydrodynamic coefficients on the heave direction can be obtained by the integration of the 
pressure on the disk hull:

$$
\begin{gathered}
f_{3}^{d}=i 2 \pi \rho g A \int_{0}^{R_{M}}\left\{\phi_{7 U}^{0}(r,-d)-\phi_{7 L}^{0}(r,-d)\right\} r d r \\
\mu_{33}+i \lambda_{33} / \omega=2 \pi \int_{0}^{R_{M}}\left\{\phi_{3 U}(r,-d)-\phi_{3 L}(r,-d)\right\} r d r
\end{gathered}
$$

\section{RESULTS}

In this section, to examine the advantage of the moon pool and optimize the wave energy conversion ability of the WEC, hydrodynamic analysis of a certain disk with several cases of moon pools is considered. As a measure of wave energy conversion ability, the magnification $\eta / \eta_{0}$ of the optimal power take-off of the disk with moon pool relative to the single disk is also calculated. In the solving process, to reduce the computational complexity and highlight the key variables, some geometrical dimensions such as $\mathrm{h}=60(\mathrm{~m}), \mathrm{e}=3(\mathrm{~m})$, $\mathrm{t}=5(\mathrm{~m}), \mathrm{b}=11(\mathrm{~m})$ and $\mathrm{R}_{\mathrm{m}}=4.0(\mathrm{~m})$ are confirmed.

As shown in Fig.2 4, the hydrodynamic coefficients in a dimensionless representation and connected wave energy conversion ability are plotted versus dimensionless wave period $\mathrm{T}^{*}=\left(\mathrm{g} / \mathrm{R}_{\mathrm{M}}\right)^{1 / 2} \mathrm{~T}\left(\mathrm{R}_{\mathrm{M}}=\right.$ disk radius, $\mathrm{T}=2 \pi / \omega=$ wave period) for varies geometries of the moon pools. No spring is assumed to be present, i.e. $\mathrm{k}=0$ as the optimal spring calculated before cannot be realized in fact. It is observed from Fig. 2 that the geometry of the moon pool has great influence on the hydrodynamic performance of the disk. The non-dimensional added mass in heave of the disk is friendly period-dependent and nearly stays the same at longer period and decrease with the increase of $\mathrm{R}_{\mathrm{T}} / \mathrm{R}_{\mathrm{M}}$.
The non-dimensional heave damping is highly perioddependent with a higher peak damping occurs at the same period for a smaller $\mathrm{R}_{\mathrm{T}} / \mathrm{R}_{\mathrm{M}}$. In addition, it will be negative when the period is long enough. The non-dimensional amplitude of excitation force in heave direction is highly dependent on wave period and approaches zero for very short and long high period. The wave periods where the peak of excitation force occurs are same and the bigger $R_{T} / R_{M}$ results in a lower peak. The wave energy absorption magnification $\eta / \eta_{0}$ is highly dependent on period with inconspicuous peak which is inversely related with the value of $R_{T} / R_{M}$.

Fig. 3 depicts the hydrodynamic coefficients of disk and energy absorption magnification with a higher $\mathrm{R}_{\mathrm{E}} / \mathrm{R}_{\mathrm{M}}=2.5$. The added mass in heave increases rapidly at short wave period and nearly stays the same at long period for a certain $\mathrm{R}_{\mathrm{T}} / \mathrm{R}_{\mathrm{M}}$, and stays nearly the same with the increase of $\mathrm{R}_{\mathrm{T}} / \mathrm{R}_{\mathrm{M}}$ at short wave period. The non-dimensional heave damping is highly dependent on period and a higher peak damping occurs at a longer wave period for a smaller $R_{T} / R_{M}$. The nondimensional amplitude of excitation force in heave direction is highly dependent on wave period and approaches zero for very short and long high period. The wave period the peak occurs and the peak decrease with the increase of $R_{T} / R_{M}$. The wave energy absorption magnification $\eta / \eta_{0}$ is highly dependent on period and approaches zero at very long period for bigger $\mathrm{R}_{\mathrm{T}} / \mathrm{R}_{\mathrm{M}}$, and null points occur for smaller $\mathrm{R}_{\mathrm{T}} / \mathrm{R}_{\mathrm{M}}$. The wave period bandwidth where the magnification is greater than one is highly dependent on the geometry of the moon pool and decrease with the increase of $R_{T}$.
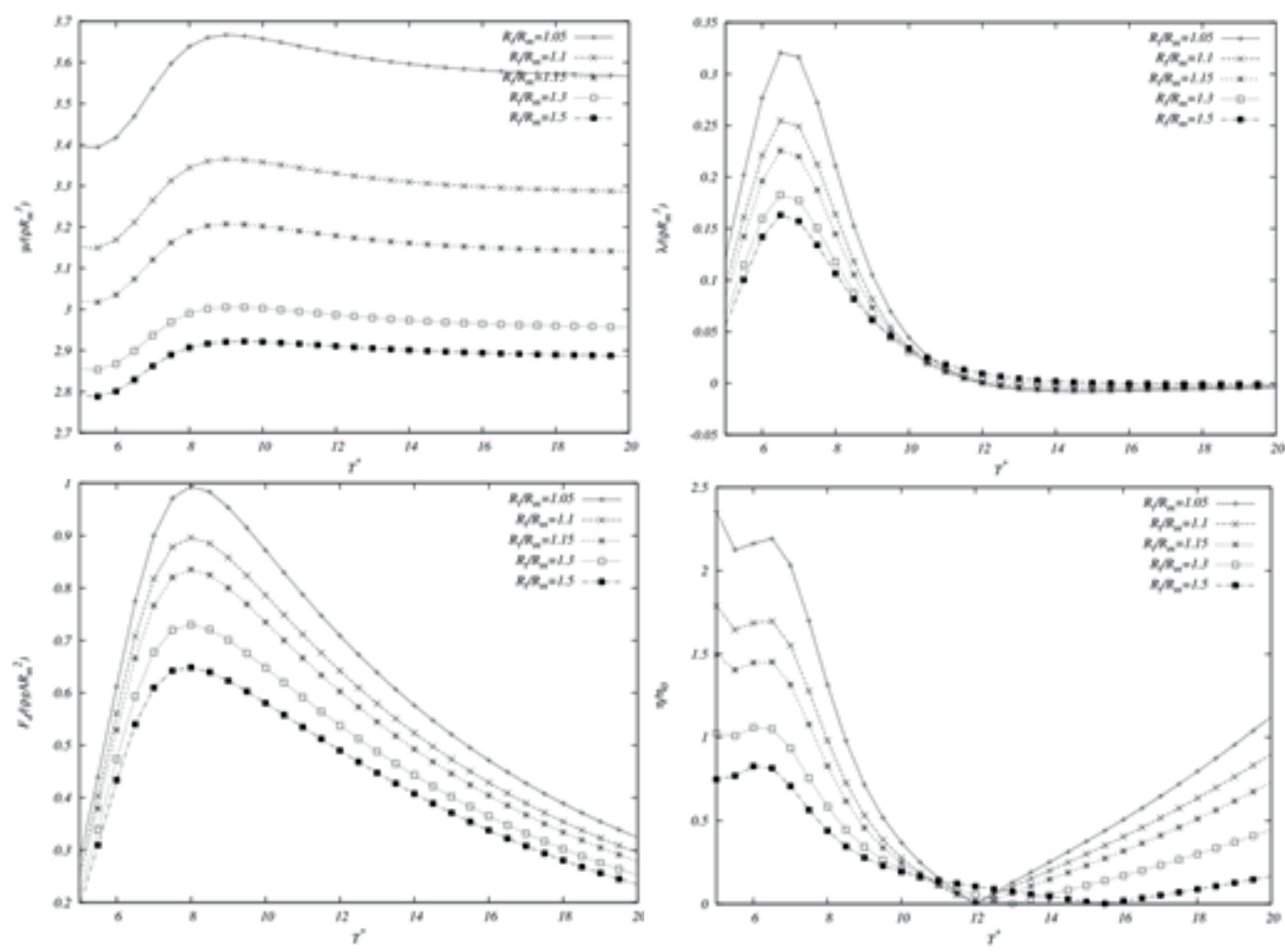

Fig. 2 Hydrodynamic analysis and energy magnification for various $R_{T} / R_{M}$ with certain $R_{E} / R_{M}=1.5$ 

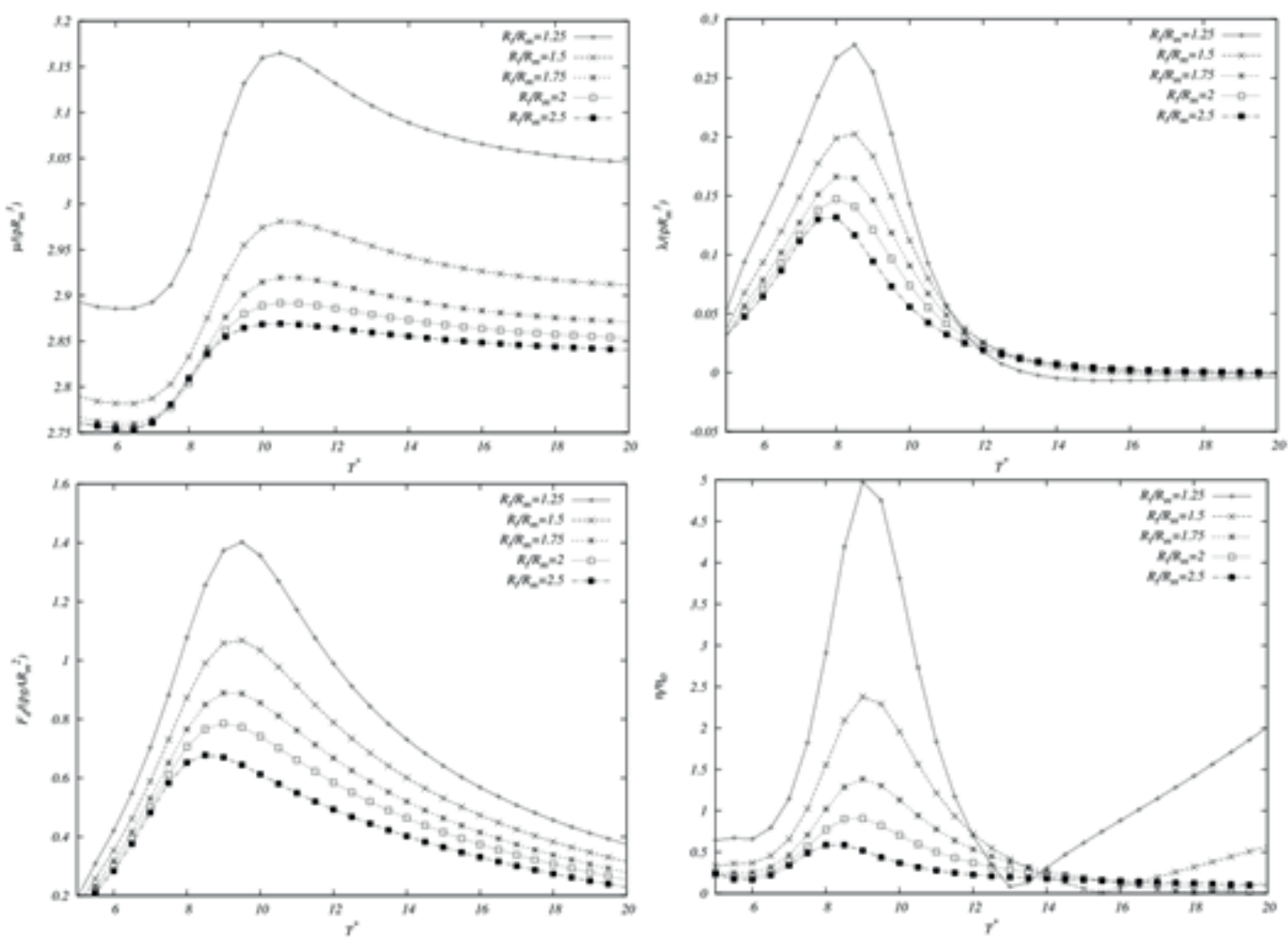

Fig. 3 Hydrodynamic analysis and energy magnification for various $R_{T} / R_{M}$ with certain $R_{E} / R_{M}=2.5$
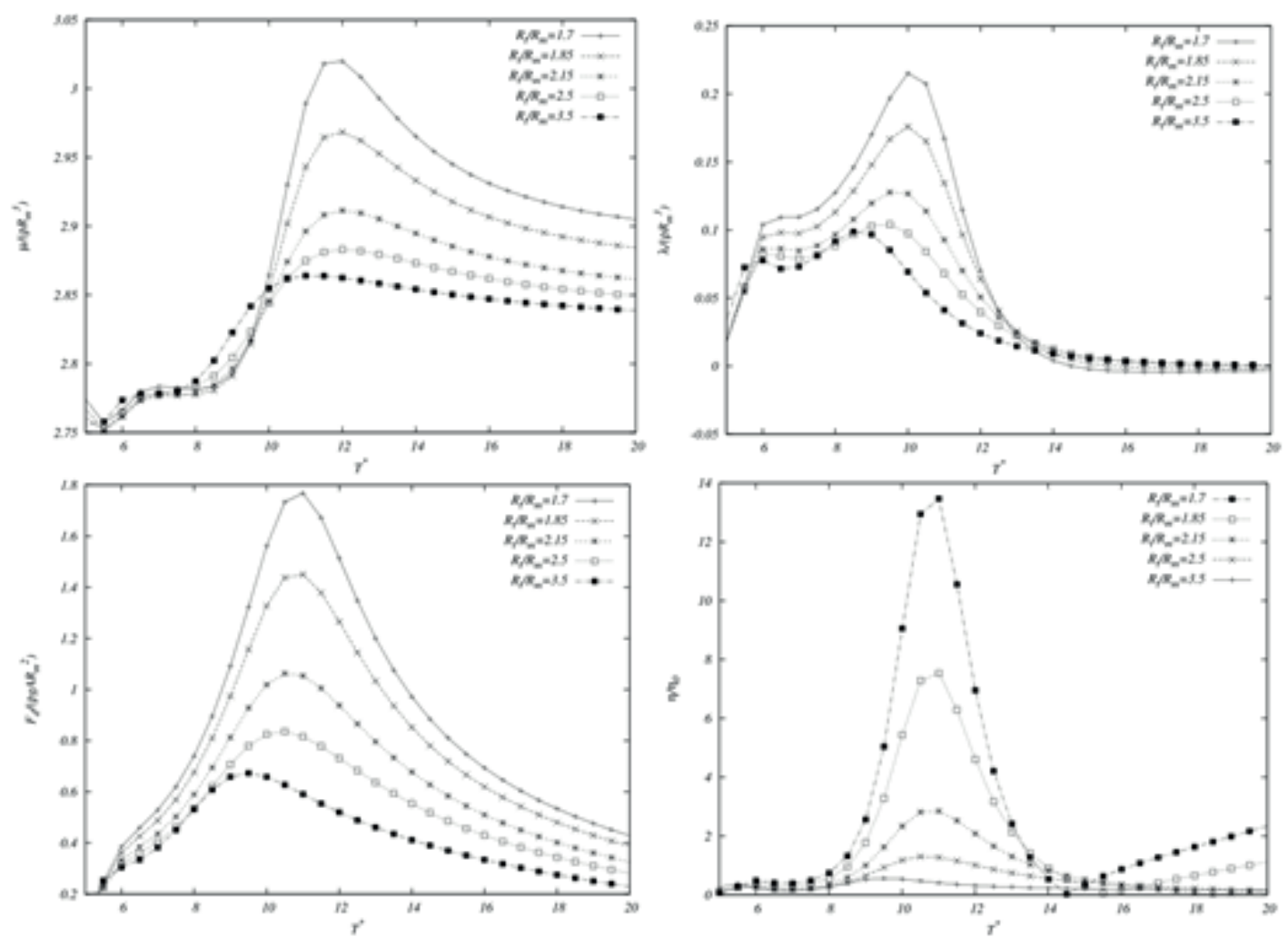

Fig. 4 Hydrodynamic analysis and energy magnification for various $R_{T} / R_{M}$ with certain $R_{E} / R_{M}=3.5$ 


\section{CONCLUSIONS}

Although the hydrodynamic frequency-domain analysis of the WECs is based on some assumptions and ignorance of some affects such as the non-linearity in this paper, the presented analytical approximation provides an efficient solution for the fundamental research. Despite the negative effect caused by the assumption, the analysis of the effects for different geometries especially for the fixed water depth is good, from which we can have a good understanding of the effects of different geometries for fixed mass on the hydrodynamic performance.

In the research for the ability of WECs for the energy conversion, a series of geometries based on the fixed water depth are considered respectively, from which we find that the geometry of the moon pool have great effect on the ability of energy conversion and when the radius of moon pool is closed to that of disk, the conversion ability will be the best.

\section{REFERENCES}

1. Budal K, Falnes J, Iversen LC, Lillebekken PM, Oltedal G, Hals, et al. (1982) The Norwegian wave-power buoy project. In: Proceedings of 2nd International Symposium on Wave Energy Utilization, Trondheim, Norway, 323-44.

2. Chen XB, Liu HX, Duan WY (2015) Semi-analytical solutions to wave diffraction of cylindrical structures with a moon pool with a restricted entrance. Journal of Engineering Mathematics, 90(1), 51-66.

3. Eriksson M, Isberg J, Leijon M (2005) Hydrodynamic modeling of a direct drive wave energy converter. Int. J. Eng. Sci., 43: 1377C1387.

4. Falcao AFO (2007) Modeling and control of oscillatingbody wave energy converters with hydraulic power takeoff and gas accumulator. Ocean Engineering, 34(14-15), 2021-2032.

5. Garrett CJR (1971) Waves forces on a circular dock. J. Fluid Mech. 46, 129-39.

6. Henderson, R (2006) Design, simulation, and testing of a novel hydraulic power take-off system for the Pelamis wave energy converter. Renewable Energy, 31(2), 271-283.

7. Liu HX, Duan WY, Chen XB (2012) Analytical solution of wave loads on cylindrical structures. Proc. ICMT2012, Harbin (China).

8. MacCamy RC, Fuchs RA (1954) Wave forces on piles: a diffraction theory. US Army Corps of Engineers, Beach Erosion Board Tech. Memo. No. 69.

9. Margheritini L, Vicinanza D, Frigaard P (2009) SSG wave energy converter: design, reliability and hydraulic performance of an innovative overtopping device. Renewable Energy, 34(5), 1371-1380.

10. Mavrakos SA, Chatjigeorgiou IK (2006) Second-order diffraction by a bottom-seated compound cylinder. Journal of Fluids and Structures 463-492.

11. Miles JW, Gilbert JF (1968) Scattering of gravity waves by a circular dock. J. Fluid Mech. 34, 783-793.

12. Price AAE, Dent CJ, Wallace AR (2015) On the capture width of wave energy converters. Applied Ocean Research, 31(4), 251-259.

13. Weinstein A, Fredrikson G, Parks MJ, Nielsen K (2004) Aqua-BUOY: the off-shore wave energy converter numerical modeling and optimization In: Proceedings of MTTS/IEEE Techno-Ocean., 04, 1854C1859, Kobe, Japan.

14. Almar, R; Almeida, P; Blenkinsopp, C; Catalan (2016), P. Surf-Swash Interactions on a Low-Tide Terraced Beach. Journal of Coastal Research. SI 75, 348-352.

15. Wu BJ, Lin HJ, You YG, Feng B, Sheng SW (2010) Study on two optimization methods of oscillating wave energy conversion device. Acta Energiae Solaris Sinica, 31(6), 769774, Chinese.

16. Yeung RW, Peiffer A, Tom N (2012) Design, analysis and evaluation of the UC-Berkeley wave-energy extractor. J. Offshore Mech. Arct., 134(2): 02190

\section{CONTACT WITH AUTHOR}

$$
\text { H.X. Liu }
$$
College of Shipbuilding Engineering, Harbin Engineering University, Harbin 150001, China

email: liuhengxu@hrbeu.edu.cn; 15045630022@139.com 